Cahiers de recherches médiévales

\title{
Poisons, sorcières et lande de bouc
}

\section{Nicolas Ghersi}

\section{OpenEdition}

Journals

Édition électronique

URL : https://journals.openedition.org/crm/11507

DOI : $10.4000 / \mathrm{crm} .11507$

ISSN : 1955-2424

Éditeur

Honoré Champion

Édition imprimée

Date de publication : 15 juin 2009

Pagination : 103-120

ISSN : 1272-9752

\section{Référence électronique}

Nicolas Ghersi, «Poisons, sorcières et lande de bouc », Cahiers de recherches médiévales [En ligne], 17 | 2009, mis en ligne le 15 juin 2012, consulté le 15 décembre 2022. URL : http:// journals.openedition.org/crm/11507 ; DOI : https://doi.org/10.4000/crm.11507

Ce document a été généré automatiquement le 15 décembre 2022.

Tous droits réservés 


\title{
Poisons, sorcières et lande de bouc
}

\author{
Nicolas Ghersi
}

1 L'histoire de la sorcellerie dans l'Occident médiéval, qui suscite tant d'articles et d'ouvrages, pose immanquablement la question de la genèse de la grande chasse aux sorcières ${ }^{1}$. Depuis les travaux de P. Paravy, de nombreux historiens, notamment autour de l'Université de Lausanne, ont repris à leur compte l'hypothèse de l'arc alpin, avec parfois moins de prudence que ceux qui l'avaient initiée. Elle présente de nombreux atouts : unité de lieu (Dauphiné, Savoie, Val d'Aoste, Valais), unité de temps (du concile de Constance au concile de Bâle), unité d'action (les premiers bûchers de sorciers coïncident avec les premiers traités de démonologie). Elle a pour mérite de distinguer démonolâtrie et sorcellerie, mais aussi de ne pas confondre les superstitions magiques et populaires, largement tolérées, du prétendu complot contre la Chrétienté, scellé lors $\mathrm{du}$ sabbat où mène un vrai ballet nocturne. Elle repose surtout sur une documentation abondante, qui fait sans conteste de ces régions un épicentre majeur du phénomène sorcellaire.

2 Cette documentation fait défaut dans le Midi, ou plutôt, ces procès épars, dans les maigres sources des registres d'officialité, de justice consulaire ou seigneuriale, parfois simplement évoqués dans une minute notariale, n'ont pas fait l'objet d'une recension exhaustive ${ }^{2}$. Les Pyrénées, longtemps présentées comme le berceau du sabbat, ont donc abandonné aux Alpes la paternité de la chasse aux sorcières, depuis que les mystifications de Lamothe-Langon ont été éventées par N. Cohn et R. Kieckhefer ${ }^{3}$. Elles constitueraient pourtant une solide alternative: le sabbat y est attesté, dans le Val d'Aneu, dès 1424 ; les premiers bûchers s'élèvent déjà depuis une vingtaine d'années, sur les pas de Vincent Ferrier; les traités d'Alfonso Tostado ou d'Alfonso de La Espina évoquent le vol nocturne ou le pacte diabolique avec des accents plus «modernes » que le Formicarius de Nider; leurs parcours croisent ceux de Jean Vineti et d'Hugues Nigri, les premiers chasseurs de sorciers.

3 La sorcellerie méridionale semble codifiée dès le début du $\mathrm{XV}^{\mathrm{e}}$ siècle, puisqu'elle suppose 1) le vol nocturne des femmes, 2) la réunion sur la montagne ou sur la lande de bouc, 3) l'hommage au diable sous forme de bouc, 4) l'osculum infâme, 5) le meurtre par poisons de petits enfants. Certains de ses traits sont originaux : la pratique conjointe du 
vénéfice et du sabbat est attestée dès que l'affaire est un peu documentée ; le crime de sorcellerie ne concerne que les femmes, sans exception; le diable apparaît sous les traits d'un bouc, à peu près inconnu dans les procès alpins. C'est à ces trois topoi, aux accents nettement modernes, puisqu'ils sont largement diffusés hors de cette aire géographique aux $\mathrm{XVI}^{\mathrm{e}}$ et $\mathrm{XVII}^{\mathrm{e}}$ siècles, et à leur articulation que nous consacrerons cette étude.

\section{Les poisons}

Faisant le point sur la contiguïté entre poison et sorcellerie, F. Collard montre que les liens entre ces deux crimes ne sont pas souvent évoqués et fournit des chiffres convaincants ${ }^{4}$. Il conclut même: "En réalité, l'immense majorité des «faits" de sorcellerie traités en justice se révèle vide d'empoisonnement ». C. Gauvard souligne la convergence féminine de la sorcellerie et de l'empoisonnement dans la littérature des $\mathrm{XIV}^{\mathrm{e}}$ et $\mathrm{XV}^{\mathrm{e}}$ siècles ${ }^{5}$ et le Roman de la Rose chante les sorcières qui servent du venin à leurs filiâtres ${ }^{6}$, mais dans les sources narratives du Moyen Âge, sur 225 affaires de poisons, 16 seulement y mêlent aussi sorcellerie ou diabolisme. Dans les sources judiciaires, la proportion est encore moins importante, puisqu'au Parlement de Paris, poison et sorcellerie sont associés 2 fois sur 22 .

5 Même s'ils sont rarement attestés au nord de la Loire, les liens entre poisons et sortilèges existent bien. Ainsi, au domicile de quatre femmes notoirement considérées comme sorcières, le prévôt de Paris retrouve des herbes et des crapauds. Pressées par les questions, les prévenues évoquent «chapeaux à fleurs, vœu de cire, poële à frire, crapauds nourris de lait maternel, autant de gestes, en particulier culinaires ou sexuels qui sont des affaires de femmes ${ }^{7}$. En Italie, en Provence, en Avignon ou dans le Lyonnais, la confusion des deux crimes reste rare, au $\mathrm{XV}^{\mathrm{e}}$ siècle, mais Jacques Chiffoleau note que «les frontières sont floues entre l'empoisonnement et l'envoûtement " ${ }^{8}$ et Nicole Gonthier parvient aux mêmes conclusions ${ }^{9}$. La proportion de sorciers-empoisonneurs sur les rives du Léman est déjà plus importante (24\%). Pourtant, à Toulouse, toutes les sorcières sont aussi et d'abord des empoisonneuses.

De la Gascogne à la Chalosse et de l'Auvergne aux Pyrénées, le poison est à ce point lié à la sorcellerie que le terme "pousouera ", avec ses variantes,a fini par désigner toutes les présumées sorcières, qu'elles usent ou non de poisons ${ }^{10}$. La confusion avec la sorcellerie est telle qu'une main posée sur une épaule, des caractes ou des formules incantatoires peuvent lui être assimilés ${ }^{11}$. Le mot "pousouera» est attesté comme synonyme de "sorcière » dans tous les dictionnaires (Mistral, Alibert, Lagarde, Lespy, Moureau, Palay, Roques, etc.), et s'est maintenu dans les langues et dialectes méridionaux. Si la sorcière du Midi est d'abord une "pousouera ", elle est parfois autrement qualifiée. Elle est aussi une "faitilhera ", c'est-à-dire tout à la fois une «femme fatale » (du latin fatum), qui a la maitrise du Destin,une «factrice » (du latin factura), faiseuse de sorts, avec des poupées de chiffon pour créatures, une "clandestine " (du latin facticius), qui appartient au monde de la nuit et des crimes furtifs, comme les empoisonneuses ou les larronnesses, une "meurtrière " (du latin facinus) qui tue de petits enfants, une "envoûteuse " (du latin fascinatio), qui use de bandelettes, amulettes et phylactères. Plus rarement, la sorcière méridionale est une " masca ", portant un masque de suie, pour le vol nocturne qu'elle accomplit depuis sa cheminée, le pied sur la crémaillère ${ }^{12}$. 
7 La combinaison des crimes de poison et de sorcellerie est donc classique dans le Midi. À Montaillou, Le Roy Ladurie note que "les savoirs magiques sont divers, et souvent, mais pas exclusivement, féminins", mais que l'inquisiteur "ne s'arrête point à ces vétilles de petite magie $»^{13}$. La veuve Béatrix de Planissoles consulte d'abord une juive convertie qui lui conseille de maculer du linge de sang menstruel pour faciliter l'amour de ses filles et de porter les cordons ombilicaux de ses petits-fils si elle veut gagner ses procès $^{14}$, puis un pèlerin qui lui donne des graines d'ive pour guérir son petit-fils de l'épilepsie et des grains d'encens contre les maux de tête ${ }^{15}$. Si cette " petite magie » et ces remèdes paysans laissent froid Jacques Fournier, ils sont bientôt poursuivis avec plus de fermeté, tant dans les vallées ariégeoises qu'en Navarre et au Pays Basque.

8 C'est en Basse-Navarre, dans la région de Labastide-Clairence, en 1328, que le baylede la châtellenie d'Ultrapuertos arrête à Bidache Johana la Christiana «qui empozonava la gent et era erbolera mala $»^{16}$. Cette guérisseuse fait peut-être partie d'un groupe de cagots, ces "lépreux sociaux ", comme les qualifie Françoise Bériac, souvent médecins, que l'on nommait « crestians » en Béarn. Elle dénonce quatre de ses complices, Arnalda del Bosc, Peyrona et Johana de Preyssac et Domenja de Durban, autres «faytilleras e erboleras ", qui sont toutes brûlées. Un an plus tard, non loin de là, à Saint-Jean-Piedde-Port, Alamana de Mearin, du pays de Mixe, est remise au châtelain par la cour de Pampelune comme "faytillera », et Jurdana de Irissari, «herbolera», est brûlée. En 1338, Condesa de Urritzaga est accusée d'avoir commis des « faytillas » par les habitants de Lasse, et elle est condamnée au bûcher à Saint-Jean-de-Port «propter sortillegia perpetrata per eam ». En 1342, la dame de la maison d'Arostéguy et une femme de Gabat sont exécutées comme sorcières à Garris. En 1370, près de Mixe, Johan Arnalt, d'Ilharre, accuse les «faytiles et faytileres » Pes de Goytie et Condesse de Beheytie de l'avoir aidé à tuer le fils de Guixon d'Eliçague, et les trois sorciers comparaissent devant Jeanne d'Évreux, la reine de Navarre. En 1377, en Lomagne, des «faxilleras »sont accusées d'oppresser les gens à Escazeaux ${ }^{17}$. En Béarn, en 1393, Mariole du Colom, de Lucq, diffamée d'être "posoere", est arrêtée par le procureur du vicomte, mais l'intervention de ses amis la sauve du bûcher, et elle est libérée.

Les habitants de Montesquiou, dans le Gers, réputés sorciers, sont encore surnommés " pousoès »au XIX ${ }^{e}$ siècle. Six siècles plus tôt, Jeannette Nova, de Saint-Chaffre, qui fabrique des poisons, est une sortilega vel facineria, elle est poursuivie pour des facinis, sortilegiis ac potionibus ${ }^{18}$. Aelips Cavalade, de Millau, use « de fachilharias, de meguianas e de poysos ${ }^{19}$. Franque de Foix et Péronne de Giniac, de Barran, dans le comté de Fezensac, sont accusées en 1447 de pocionibus, fachileriis et sortillegiis. En 1470, les jurats d'Oloron jugent une "posoere ", coupable de sortilèges. Monet d'Ondsart, d'Aroue, en Basse-Navarre, est " pousoer et faitillier »; Goalhardine de Bertretexs use de l'art " de posoerie et fatilherie "; Audine de Mimbiela, d'Arthez, est convaincue de pratiquer «pozoeria et faytilharia ». En 1520, un article de la coutume de Bordeaux punit de mort tout homicide « am faytilhas o am poyssos ». Cette énumération fastidieuse souligne les liens du poison et du sortilège, mais c'est aussi une opposition récurrente, fixée dans le lexique, qui semble témoigner que la "faitilhera", loin de se confondre avec l'empoisonneuse, est la marque d'une autre activité des sorcières, et que le vénéfice est donc un simple artifice parmi d'autres.

10 Les registres du Parlement de Toulouse, pourtant tenus en langue d'oïl, ignorent à peu près le mot "sorcière », préférant parler de "pousonière ». Avec le vocabulaire de la trahison, du larcin et de la lèpre, le mot concurrence les invectives sexuées dans 
l'arsenal des injures en vogue à la fin du $\mathrm{XV}^{\mathrm{e}}$ siècle. Ainsi, lors d'une rixe qui oppose Antoine de Giéré, seigneur de La Motte, au receveur du feu comte d'Armagnac, celui-ci l'insulte et le traite de "ribault, barataire, filz de pousonniere $»^{20}$. Une des sorcières de Millau, Garine est interrogée comme " pousoniera et sortilleria ${ }^{21}$. Chateta, une des dix-huit sorcières de Marmande, accuse Péronne de Belleville d'être "pousonnière " ${ }^{22}$. Quand Jeannette Romat, leur complice, est sur l'échafaud, «le baile l'amena devant ladite Péronne, laquelle confessa qu'elle estoit pousonniere XXX ans avoit, et avoit mieulx gaigné estre brulée que l'autre, et de soy mesme monta sur le chaffault, le baile veiant cela ordonna qu'elle feust brulée et commanda que on alast querir des fagotz ${ }^{23}$. Dans les prisons du Parlement de Toulouse, une femme « diffamée d'estre sourciere et pousonniere ", est violée par Vincent Marin ${ }^{24}$. Ce Marin, qu'on dit hérétique, vivait avec trois femmes et en a avorté deux autres, avec des ampoules ardentes et des «ymages »; il a aussi rompu les vœux de chasteté d'une religieuse, commettant ainsi un inceste ${ }^{25}$. En quarante ans d'activité parlementaire, c'est là la seule rencontre, fortuite, que l'on relève entre le petit monde des magiciens et celui des sorcières et des empoisonneuses. En Gascogne, Languedoc, Quercy et Rouergue, toute affaire un peu documentée évoque ces sorcières-empoisonneuses, alors que les confins du Parlement, en Vivarais ou en Velay, les ignorent, ce qui confirme la piste pyrénéenne ${ }^{26}$.

11 Ce sont les terres que le comte d'Armagnac conteste tout au long du XV siècle aux agents du roi, et surtout la frontière d'Astarac et de Comminges, qui connaissent de brusques accès de fièvre, chaque fois que l'arrogante maison connaît la disgrâce. La géographie du vénéfice et de la sorcellerie épouse donc ces zones de conflits, traversées par les routiers, et la chronologie des bûchers en jalonne les disputes. De Casamajour, avocat des sorcières d'Orleix, précise « que en Bigorre s'est levé bruyt contre les vieilles femmes et leur a l'en imposé qu'elles sont toutes pousonnieres $\aleph^{27}$. Les mêmes rumeurs courent la Gascogne: "on dit que en Gascoigne a grant habondance de pousonnieres $\aleph^{28}$. Antoine Setgier, avocat de Gailhardine, épouse d'Arnault de Dailhet, habitant Auch, la présente comme une «bonne pouvre femme et mariée, a tousjours bien vesquu et son mary est bien content d'elle, ce nonobstant, au pourchaz de quelques ungs ses malvueillans qui luy ont imposé qu'elle estoit pousonniere ${ }^{29}$. Lors $\mathrm{du}$ procès des sorcières de Rieumes, Pierre Suaire se désolidarise des autres consuls, mais Morilhon, l'avocat de ses pairs, prétend qu'il est parent d'une des "pousonnieres» et qu'il est leur complice ${ }^{30}$. À Castelnau-Picampeau, une femme nommée Bertrande, accusée par les consuls et leur assesseur, Noailles, d'être "pousonniere et devine », dénonce Jeanne de Serracave avant d'être brûlée ${ }^{31}$. Deux ans plus tôt, ses voisines de Montespan et de Cazères, Maria de Clauso, Peronna de La Fage et La Bascoada " estoient grandes pousonieres et avoient donné beuvrages à aucunes gens qui s'estoient prins à bayer comme les chiens ». Ce pays de Savès avait déjà connu pareil scandale : « vray est que es parties par delà souvent amennent semblables cas " $^{32}$. La contiguïté entre poisons et sortilèges est entretenue par une longue tradition juridique, mais l'amalgame du veneficium et du maleficium repose aussi sur des éléments concrets, ou jugés tels. Comme Esclarmonde Gausagne, sorcière de Laroque-d'Olmes, qui dissimule son poison dans un drapelet puant (1447), une des sorcières de Seix a son poison « tout plié avec ung petit drap de lin attaché avec ung filliet noyr » (1561), et les lépreux de Languedoc et de Gascogne ont reçu un petit sac de cuir ou de drap contenant le poison destiné à infecter les puits $(1321)^{33}$. Si le poison des empoisonneuses est le réalgar, le brouet des sorcières mêle le bestiaire maléfique aux herbes odorantes, et les « pousoueras »sont d'abord des « erboleras », des guérisseuses, 
telle Catherine Coteric, qui prépare un emplâtre d'herbes pour Catherine Genoyn, piétinée par un revenant, ou Gailhardine de Dailhet, qui administre un contrepoison dans la soupe de Jamet de Fossio ${ }^{34}$. Pierre Fabre et Raimond Cathalan, en 1330, précisent que ces herbes étaient ramassées à la Saint-Jean, ce que confirme Péronne Bachême, la sorcière de Labruguière ${ }^{35}$. Des procès postérieurs, comme celui des sorcières ariégeoises de Seix en 1561 ou les procès de Pierre de Lancre en 1613-1614 assurent que le calendrier des sorcières méridionales correspond à ce qui a été relevé ailleurs en Europe : $1^{\text {er }}$ mai, $1^{\text {er }}$ novembre et fête de la Saint-Jean forment trois temps forts de leurs activités.

13 À ces remèdes de bonne fame fait écho une cuisine autrement répugnante et savamment toxique, qui rompt les usages de la commensalité. Si le bouc préside au sabbat, le crapaud accommode tous les plats : les lépreux des basses-vallées ariégeoises (1320), Guillerma Robberta de Gourdon (1320), Pierre Ricord de Béziers (1329), Bernard Fort de Cordes (1344) ou Esclarmonde Gausagne (1447) y ont recours, comme Margot la Quesnote, une autre envoûteuse, qui en 1380 avait porté un gros crapaud dans un drap blanc $^{36}$, ou Mathe de Ga, la sorcière ariégeoise, qui a nourri pendant deux ans un crapauddans un trou de sa maison ${ }^{37}$. Les onze sorcières de Montespan et de Cazères préparent leurs poisons à base de fiel de grenouilles et de tortues, de cervelle de chat et de langue de chien enragés ${ }^{38}$. Deux chats noirs, esprits malins qui ont peut-être donné leur nom aux Cathares, font la veillée funèbre de l'inquisiteur de Carcassonne, Geoffroy d'Ablis ${ }^{39}$. La cervelle de chat permet à Michelle Pironne, en 1469, de se débarrasser d'un jeune homme en le rendant fou ${ }^{40}$. Outre le crapaud, les autres reptiles tiennent une place importante dans la faune satanique. Guillaume Agasse, le lépreux de Pamiers, prépare ses poisons avec des "poudres de crapaud diaboliques", de l'hostie consacrée, des serpents, des orvets, des lézards, des chauves-souris et des excréments. À Gourdon, les sorcières relèvent leur potage d'ongles (onychomancie) et d'ossements d'enfants pulvérisés. À Millau, les Montcalm, médecins du comte d'Armagnac, servent aux Cornilh un "ortocreon de scaramides " (une tourte d'artichauts aux écrevisses), et Gailhardine de Dailhet prépare une fougasse farcie de chenilles et trempée dans le vin pour sa rivale Jeanne du $\operatorname{Pin}^{41}$. La cuisine des sorcières du Midi semble méconnaître brouet d'araignées, fourmis et chouettes, pourtant prisées et attestées à Paris, mais c'est là simple effet de sources: les fourmis de Jean Nider nichent aussi sous les fondations de la maison de Bernard Fort, le sorcier de Cordes, et deux chouettes sont venues hululer sur le toit de sa maison, après la mort de Na Roque, Brune Pourcel de Montaillou comprenant que le Diable l'emportait ${ }^{42}$.

Le recours aux vénéfices répond souvent, chez les sorcières, aux mêmes mobiles que ceux qui animent les empoisonneuses épargnées par le démon ${ }^{43}$. Les «lovendrincs " miraculeux facilitent la séduction; les potions abortives ou celles qui rendent féconde permettent de contrôler les naissances; les breuvages toxiques mettent un terme aux adultères et aux violences conjugales, celle qui se débarrasse de son mari tombant sous le coup de la terrible loi du parricide, menacée d'être jetée dans un sac en pleine mer, avec un singe, un serpent, un coq et un chien ${ }^{44}$. Comme les ensorceleuses agissent per industriam et per insidiam, l'axiomatique de l'intérêt peut dicter leur conduite; elles partagent cette infamie avec les larronnesses, dans ce «monde de l'ombre et de la dissimulation » (V. Toureille), pour capter plus tôt les héritages.

Mais le poison occupe une place parfois importante dans le rituel des sorcières. Dans le procès des empoisonneuses de Cazères (1475), il est question du « mal de layra », forme 
d'hystérie ou de crises d'épilepsie collective caractérisée par des aboiements, qui sévit parfois au Pays Basque et que décrit François Bordes à propos des sorcières d'Amou (1613), tandis que la figure du loup-garou est bien connue dans les Landes ${ }^{45}$. Enfin, le poison leur sert aussi à l'occasion du sabbat, en deux circonstances : elles en oignent le bâton qui les mène au sabbat et l'administrent aux petits enfants qui sont sacrifiés au bouc de Biterne ${ }^{46}$.

\section{Les sorcières} seulement 6 sorciers. Si l'on élargit la région au Pays Basque, aux Landes et au Béarn, et qu'on tient compte des quelques procès évoqués par d'autres sources, on obtient une proportion comparable : 11 sorciers pour 145 sorcières. Le siècle précédent offre un déséquilibre identique : 3 sorciers pour 20 sorcières ${ }^{47}$. Encore faut-il voir qui sont ces « sorciers »: Jean Garin, menacé en 1437 à Millau, n'est plus poursuivi sept ans plus tard; le procès de Barthélémy Vidal, le "sourcier» de Vauvert, est renvoyé du Parlement sans aucun détail ; l'autre vieillard, Jean Salarde, n'est pas arrêté ; les deux hommes de Désaignes ne sont que des « complices »; quant à Pierre Vallin, condamné au bûcher en 1438, et Philippe Calvet, le sorcier toulousain, ils sont jugés à Vienne et à Paris, hors des limites du Parlement de Toulouse. À Léran, l'avocat du roi, dans sa plaidoirie, parle d'abord d'un sorcier et de deux sorcières, accordant d'ailleurs le mot de "pouçonnier " au féminin, comme si la présence d'un homme était incongrue ${ }^{48}$. Puis au cours du même procès, le trio s'est réduit à deux femmes ${ }^{49}$, avant que ces femmes n'en devinssent trois ${ }^{50}$ : le processus de féminisation de la sorcellerie méridionale était achevé.

Entre 1424 et 1520, sur 351 personnes examinées pour un délit de sorcellerie en Dauphiné, $70 \%$ sont des femmes. Pourtant, dans la période précédente (1350-1415), il y a autant de sorciers que de sorcières poursuivis en Dauphiné. La féminisation semble moins marquée en Forêt Noire, en Souabe, dans les vallées vaudoises et valaisannes, dans les cantons de Fribourg, de Berne ou du Tyrol, mais elle s'accroît par la suite. Les procès francs-comtois ou bourguignons du milieu $d u \mathrm{XV}^{\mathrm{e}}$ siècle sont à dominante masculine, comme la Grande Vauderie d'Arras. Les 97 lettres de rémission ${ }^{51}$ et les arrêts du Parlement de Paris présentent autant de sorciers que de sorcières, sauf en ce qui concerne la magie amoureuse, mais l'épave du registre criminel du Châtelet (1390-1391) signale peut-être des évolutions contradictoires dans cette répartition ${ }^{52}$.

De ce point de vue, la sorcellerie méridionale est donc tout à fait atypique. Dès le XIV ${ }^{e}$ siècle, le crime de sorcellerie semble exclusivement féminin. Ce trait caractéristique se confirme d'ailleurs par les 54 exécutions de sorcières du comté de Foix $^{53}$, comme par les procès espagnols, largement postérieurs, ou par la «chasse aux sorcières » menée au Pays Basque par Pierre de Lancre. Il serait pourtant hasardeux d'évoquer une société méridionale masculine et patriarcale, au pays du droit savant, des troubadours et des Bonnes Femmes. Le régime de la dot, les règles de succession ou la personnalité juridique des femmes contredisent l'image de la fille à marier, de l'épouse soumise, de la veuve misérable. L'homilétique misogyne de Vincent Ferrier, les œuvres littéraires de Martin Le Franc et de Jaume Roig, Champion des Dames et Livre des Dames, le regard du Parlement sur les femmes, blasphématrices impénitentes, imbéciles, perverses, frivoles, folâtres et volages donnent un cadre à nos procès, mais la

Cahiers de recherches médiévales, 17 | 2009 
littérature européenne $\mathrm{du} \mathrm{XV}^{\mathrm{e}}$ siècle toute entière colporte les mêmes antiennes et l'argument ne tient guère, puisque la féminisation de la sorcellerie dans le Midi a un caractère d'exception.

Parturientes ou sages-femmes, les femmes donnent la vie ; elles font aussi la toilette des morts. La Lex Julia de fundo dotali et les clauses de garantie des contrats de mariage permettent aux veuves d'occuper la place des hommes. Pierre Bonnassie, décrivant le droit du Midi, souligne d'ailleurs sa "tonalité féminine », tandis que Paul Ourliac et Jean-Louis Gazzaniga écrivent: «Dans tout le Midi (...), la femme possède une plus grande liberté, son père et ses frères interviennent souvent en sa faveur; les règles $d u$ régime dotal lui assurent une certaine indépendance; elle conserve en principe la jouissance des paraphernaux et (...) son mari, qui devrait payer les frais de ses obsèques, a tout intérêt à la bien traiter $»^{54}$.

L'affaire de Léran offre un prisme intéressant pour comprendre la féminisation des sorciers-empoisonneurs. L'histoire des sorcières de Léran nous est connue grâce à une procédure judiciaire qui est, sans conteste, l'affaire la plus compliquée, la plus longue et la mieux documentée du Parlement de Toulouse au XV ${ }^{e}$ siècle $^{55}$. Les Lévis étaient, avec les Armagnacs ou les Albrets, l'une des plus grandes familles du Midi. Les deux plus nobles chevaliers de la famille, Philippe I de Lévis-Mirepoix et Philippe de LévisFlorensac, sont du « voyage de Tartas » en mai 1442, auprès du roi de France. Ils ont participé à la prise de Saint-Sever. En juillet 1442, Philippe de Lévis-Florensac tombe au siège de Dax, victime d'un trait d'arbalète. Son cousin et beau-frère, Philippe de LévisMirepoix, meurt quelques semaines plus tard, à son retour au château de Lagarde, près de Mirepoix. Aelips de Lévis, une beauté du diable ${ }^{56}$, sœur de Lévis-Florensac et épouse de Lévis-Mirepoix, se trouve alors engagée dans deux procès interminables. LévisFlorensac n'a laissé qu'une fille, Jeanne, dont la tutelle fait l'objet de querelles, et qui finit par être enlevée. Lévis-Mirepoix, quant à lui, mourait sans enfant, ne laissant qu'un jeune frère, Jean IV de Lévis, qui dispute à sa belle-soeur les terres de Mirepoix. En 1446, alors que le procès au Parlement de Toulouse vient de commencer, il accuse Aelips de Lévis d'avoir empoisonné son père avec l'aide de sorcières. Voici ce qui ressort des différents témoignages.

21 Philippe de Lévis-Mirepoix a fait le guet devant Dax, sous une pluie battante qui l'a rendu malade comme beaucoup d'autres. Abattu par le décès de son cousin, il reste trois jours à Dax avec le "mal chaut» et une "fievre pestilencial», incapable de monter à cheval et mourant de soif. Il demande son congé au roi et part pour Auch, mais en chemin, il est frappé de dysenterie, et forcé de faire halte à chaque lieue. Son calvaire le mène à Mazères-sur-Salat, pour éviter les terres du comte d'Armagnac, où réside l'archevêque d'Auch, son oncle. Il y reste trois ou quatre jours, puis repart, avec Antoine d'Ortobenc pour escorte et compagnie. Arrivé à Toulouse, porte Saint-Cyprien, il doit parlementer pour qu'on lui ouvre les portes de la ville. De Toulouse, il reprend la route et met deux ou trois jours pour gagner Mirepoix, pourtant distante de dix lieues seulement. Il s'arrête en route pour dîner à Montgiscard, où on le voit jouer de la flûte.

À son retour au château de Lagarde, à la nuit tombée, son épouse l'attend. Le lendemain matin, elle lui sert à manger, mais il se contente de boire, et malgré la maladie, part à la chasse. Il fait une rechute. Les trois jours suivants, il ne quitte plus son lit. Aelips et Gausserand de Maillorque, un Catalan «de mauvaise vie », banni du royaume d'Aragon, à qui Philippe avait confié le "gouvernement de son hostel», envoient chercher un médecin catalan de Carcassonne, maître Barthélémy ${ }^{57}$. Le médecin diagnostique la 
dysenterie et repart. Les pages font venir un prêtre connaisseur de poisons qui lit dans l'urine de Philippe. La maladie de Philippe de Lévis s'aggrave. Maître Barthélémy revient ; Antoine d'Ortobenc et Aelips de Lévis restent à son chevet. Mais rien n'y fait et il meurt quinze jours après son retour, en l'absence de son frère, toujours à la guerre.

Le jour de l'enterrement, Aelips reste au lit, et un proche parent, Philippe de Voisins, seigneur d'Arques, lui rend visite et ne la trouve pas très endeuillée. La rumeur court qu'elle a empoisonné son époux ${ }^{58}$. À son retour, Jean de Lévis trouve Aelips avec Gausserand de Maillorque, marié à une "jeune demoiselle» de Mirepoix, mais on chuchote qu'il a une liaison avec la châtelaine. On raconte aussi que Thodette de Verniolle, demoiselle d'Aelips, a donné un bolet "avec de la pouldre " à manger à Philippe II, et qu'il s'est mis à vomir, est tombé malade et en est mort.

Jean de Lévis chasse Aelips et Eustache de Lévis-Florensac, évêque de Mirepoix, qui partent à Lavelanet. Il va se plaindre au roi, obtient des lettres pour faire ouvrir une enquête, mais l'huissier refuse d'arrêter Aelips et préfère envoyer ses informations au Parlement de Paris. Parmi les témoins, Bernard Carême, de Mirepoix, prétend qu'Aelips s'était renseignée sur les moyens de se débarrasser de son mari. Messire Guillaume Bonal, vicaire de l'évêque, a participé à l'extradition de Maillorque et avoue qu'il a peur qu'Aelips « le feist morir comme elle avoit fait son mary». Si l'on en croit l'avocat de Jean de Lévis, le mari de Thodette a été pris à Béziers, pour avoir empoisonné son frère, sa sœur et trois petits enfants, et alors qu'il était prisonnier et condamné à mort, il a accusé son épouse de lui avoir procuré des poisons et d'avoir aussi empoisonné le seigneur de Mirepoix ${ }^{59}$. Thodette a donc été emmenée et mise en prison. Elle fait alors appel au Parlement et est incarcérée en la Conciergerie le 17 décembre 1445. Yvonnet de Noérieux, huissier du Parlement de Toulouse, est alors chargé par la cour d'aller chercher Aelips de Mirepoix. Pendant qu'il est là, Jean de Lévis assiège le château de Mazerettes avec 300 hommes. Il s'en empare et prétend avoir trouvé dans le château un coffret rempli de poudre. Le 20 décembre, de retour de Mazerettes, sans Aelips, Noérieux se voit intimer l'ordre par la cour de faire ajourner Aelips « à son de trompete et cry publique par les lieux où elle a acoustumé de converser ». La cour menace aussi de la juger par contumace des crimes dont elle est accusée. Les biens d'Aelips de Lévis sont mis à la main du roi.

Extradé à la demande de l'évêque de Mirepoix, Maillorque est pendu et mis en quatre quartiers à Perpignan, pour d'autres meurtres. Le 19 mars 1446, la cour ordonne l'incarcération d'un frère cordelier du couvent de Mirepoix, Raoulet de Villiers, qui est accusé par Aelips d'avoir écrit des lettres closes la diffamant, mais il s'évade bientôt. Le 21 juillet 1446, un arrêt condamne Jean de Lévis à payer 4000 écus d'or pour ses injures et absout Aelips de toute poursuite. Celle-ci garde les terres de Lavelanet, Laroqued'Olmes, Ventenac et Montségur. Le 31 janvier 1447, son avocat requiert contre Jean de Lévis une amende honorable à genoux, "dessaint » et sans chaperon en la cour, à Mirepoix, à Narbonne et à Paris, et une amende profitable de 50000 écus, pour les diffamations subies.

Le procès paraissait s'enliser; il rebondit en mai 1447. Esclarmonde Sarrailh, dite Gausagne, habitant Laroque-d'Olmes, est l'épouse d'Antoine Tholosan, un Catalan qui est venu à Mirepoix servir les Lévis, avec Gausserand de Maillorque, et qui aurait fui la justice tandis qu'il était convaincu d'homicide. Son avocat la dit naïve : « est ignoscente autant que ung enfant qui nest, tant est de simple complection ». Arnaud de Casalet, bayle de Mirepoix, la dénonce à Jean de Lévis comme " poisonniere ». Chez la Gausagne 
et Tholosan vit Mondete, la cousine de Pierre-Jean Sartre, qui promet à Casalet de déposer contre la Gausagne, si on le paie bien. Il déclare que la Gausagne lui a passé la main sur le visage et l'a ainsi empoisonné. Il demande à Mondete de chercher les poisons chez sa maîtresse, un jour où Tholosan est à la foire, et lui promet «ung aguiller et une crespine». Mondete trouve sous le lit de la Gausagne une «esquille roillée close d'un drapelet, que on disoit que puoit toute ». Elle porte l'esquille à Sartre, qui la confie à Casalet. Casalet fait assembler des gens et crie : «Venez veoir les poisons de la Gausagne! ». Dans le drapelet, Casalet trouve deux cents vieux écus selon les uns (Gausagne), un peu de liqueur puante, des grains de froment, onze écus, un noble et un timbre selon les autres (Lévis).

La Gausagne est élargie per villam de Carcassonne, à caution de 500 livres. Casalet est mécontent. Il apprend que le seigneur de Léran, Gaston IV de Lévis, a pris trois "pouçonnieres". Jean de Lévis va trouver ce seigneur pour lui proposer un coup monté, et lui demande d'arrêter la Gausagne : « et luy dist qu'il failloit qu'il lui donnast territoire, disant que l'appellant estoit des fantausmes $»^{60}$. Léran demande au sorcier et aux deux sorcières d'accuser la Gausagne d'être de leur « compaignie ». Pour qu'ils la reconnaissent, puisqu'ils ne l'ont jamais vue, il dit qu'elle sera vêtue de rouge. Jean de Lévis demande à la Gausagne d'aller à Léran, et " afin qu'elle semblast femme de bien ", de porter sa robe rouge. Le sorcier et les sorcières accusent la Gausagne d'être «la maistresse qui faisoit les fantausmes et les onguemens dont elles se oignoient ». Casalet rentre à Mirepoix et confisque les biens de la Gausagne.

Jean de Lévis propose alors à la Gausagne un marché : il la délivre si elle avoue avoir fait les poisons dont est mort Philippe. Elle refuse. Il menace de lui « rompre les os à la corde de la torture ", et elle finit par céder. Alors que Jean de Lévis est à la chasse, Antoine Tholosan va trouver sa femme, lui parle à travers une grille, et elle lui dit qu'elle l'a compromis. Tholosan part à Toulouse, y trouve Eustache de Lévis, évêque de Mirepoix, et ils font appel ${ }^{61}$. Sur la place de Mirepoix, devant le peuple assemblé, la Gausagne avoue avoir donné les poisons, préparés avec « une teste de crapault et autres choses de mauvaise recepte ", à Maillorque et à Aelips, qui s'en sont servis contre Philippe. Elle ajoute qu'Aelips et Thodette sont venues à Laroque-d'Olmes chercher le poison, et que Thodette l'a mis sur un bolet ou « scamperol» (champignon). Après ses aveux, la Gausagne retourne en prison, puis maitre Jacques Gencian, conseiller du roi au Parlement, demande qu'on la mène en la Conciergerie. Pendant le trajet, sitôt franchi le pont de Mirepoix, elle revient sur ses aveux, et dit à l'huissier Jean Huet qu'on les lui a extorqués. Avec Huet, il y a maître Pierre Portier, procureur de l'évêque Eustache de Mirepoix, qui, pendant le voyage, au détour d'un buisson, aurait demandé à la Gausagne de tout nier pour éviter le bûcher.

29 Le 15 mai 1447, la Gausagne est prisonnière en la Conciergerie et subit la question. Le mardi 13 juin 1447, l'avocat dit que la Gausagne «est diffamée notoirement et publiquement XXV ans a au pais de par-delà d'estre sorciere et poisonniere et de aler avec les autres sorcieres tuer les enfans et faire autres maulx ». Il ajoute qu'il y eut des enquêtes ont été menées sur ces rumeurs du temps du feu seigneur de Mirepoix ${ }^{62}$, mais que les rapports ont été perdus, et «que la chose pululoit fort au pais des dictes sorceries et empoisonnemens"

30 L'avocat de Jean de Lévis précise que les sorcières ont un spirictum fitonicum (un esprit pythonisse) ${ }^{63}$. Aelips de Lévis embrassait Esclarmonde « et ne baisoit point les autres femmes et le dit Tholsan estoit compaignon et grant amy du dit Maillorque et estoient 
d'un mesme pais ». Il rapporte que les deux sorcières de Léran « furent interroguées et inter cetera de complicibus, et l'une apres l'autre, disdrent que la Gausagne de La Roque d'Olmes estoit la maistresse de faire les poisons de tous le pais et du bouc de Biterne et de toutes les autres meschanteries que font les sorcieres, et l'une dist que la dite Gausagne lui avoit confessé qu'elle avoit fait les poisons dont estoit mort le feu seigneur de Mirepoix, et l'autre dist et confessa pareillement ».

31 Aelips de Lévis, comme Isabelle de Ferréol ou Aygline de Montcalm, a été poursuivie parce qu'elle usurpait l'honneur des hommes. Un demi-siècle d'histoire sociale s'est privé des femmes, les rivant à la sphère privée, sous couvert d'anecdotes, ou pour en souligner le caractère d'exception, dans une historiographie "compensatrice » et « spectaculaire » comme la qualifient lucidement et rageusement Joan Kelly et Angela Lucas $^{64}$. Pourtant, la femme méridionale est partout, prenant en main le calcul économique, les échéances et les contrats, et intervenant dans la vie publique. Aux XIV ${ }^{\mathrm{e}}$ et $\mathrm{XV}^{\mathrm{e}}$ siècles, quand le mari est parti à la guerre ou sur les foires, ou quand il est décédé, la femme accapare cet honneur si largement démocratisé. Celles qu'on appelle "femmes d'honneur», femmes de bourgeois ou d'officiers royaux, peuvent éventuellement se rendre coupables d'adultère ou de comportements déviants sans perdre leur place parmi les élites. Déjà en concurrence avec la vieille noblesse, la bourgeoisie d'affaires et les gens de justice supportent de moins en moins d'obéir aux dames de l'aristocratie. La seconde moitié du $\mathrm{XV}^{\mathrm{e}}$ siècle voit ainsi se dresser les premiers bûchers de sorcières, tandis que la prostitution libre et l'adultère féminin sont sévèrement réprimés ${ }^{65}$.

\section{La « lande de bouc»}

Le crime de sorcellerie a opéré, pendant la guerre de Cent Ans, deux profondes mutations, de la démonolâtrie au vénéfice, et du vénéfice au sabbat, tandis qu'il se féminisait. Cette évolution explique que les années 1340-1430 comptent si peu de procès de sorcières, puisqu'elles sont alors d'abord des empoisonneuses. En 1408, dans le Val d'Aneu, aux marches occidentales de la Catalogne, des ordonnances prévoient des pénalités pour les gens qui rendent hommage au «boch de Biterna ", cérémonies par lesquelles ils deviennent sorciers et sorcières ${ }^{66}$. Lerida connaît alors le passage de Vincent Ferrier, dont l'eschatologie misogyne draine des foules considérables. Deux importantes chasses aux sorcières sont organisées dans cette vallée en 1419 et 1424 . Lors des procès de 1424, ces sorcières confessent se rendre de nuit au «boch de Biterna ", le reconnaitre comme seigneur, lui rendre hommage, renoncer au nom de Dieu, puis arracher de nuit les enfants à leurs mères, et les tuer par poison ${ }^{67}$.

Depuis les travaux de Saroïhandy, Barandiaran et Caro Baroja, une abondante littérature historique, mais surtout anthropologique, situe les origines de la « brujeria » espagnole dans le val d'Aneu. Parmi les travaux récents, il convient de citer ceux d'Angel Gari Lacruz, qui évoque un culte de la déesse-mère dans les grottes d'Aragon et soulignent la précocité de la sorcellerie dans les Pyrénées centrales et dans la province de Teruel ${ }^{68}$. Pourtant, pour l'anthropologue Carmelo Lison Tolosana, l'origine du « boch de Biterna » est nord-pyrénéenne. Alfonso de La Espina (cc. 1420-cc. 1495), franciscain et prédicateur itinérant, auteur du Fortalitium Fidei, déplore que les femmes perverses de Gascogne et de Dauphiné qui viennent de nuit dans des plaines désertes adorer une chèvre qui vit dans une grotte appelée le «boch de Biterna », en lui baisant l'anus et en 
portant des chandelles ardentes. Il a vécu chez l'inquisiteur toulousain Hugues Nigri vers le milieu du $\mathrm{XV}^{\mathrm{e}}$ siècle, et a pu y admirer les fresques montrant le vol nocturne et l'hommage au bouc. Or nous savons, grâce au procès d'Aygline Michel, que l'Inquisition toulousaine avait jugé des femmes pratiquant le vol nocturne et rendant hommage au diable un peu avant $1444^{69}$, et qu'à Millau aussi, comme à Mirepoix, les femmes adoraient le bouc de Biterne ${ }^{70}$.

C'est la réalité du vol nocturne qui marque la naissance du sabbat: « la grande chasse aux sorcières ne prit des proportions massives qu'avec la reconnaissance par les autorités de la réalité des voyages nocturnes $»^{71}$. En effet, si on n'admet pas la réalité du ballet nocturne des sorcières, il est impossible de croire aux gigantesques réunions où elles rendent hommage au Diable, et donc impensable d'imaginer qu'une secte de sorcières conspire contre la Chrétienté. Quinze ans plus tard, le vol nocturne est attesté dans plusieurs procès. En 1450, à Juxue, en Basse-Navarre, Johannette, dame de la maison de Sala, est arrêtée par le bayle d'Ostabaret, qui la mène à Mixe, un haut lieu de la sorcellerie. Torturée, elle avoue son affiliation à la secte satanique et sa participation aux assemblées nocturnes. «Initiée par Condeix d'Eyerabide, qui la toucha au visage et aux mains, elle se rendit plusieurs fois au sabbat grâce à une poudre dont elle se frotta les pieds ; grimpant le long du conduit de sa cheminée, elle s'envola pour rejoindre ses

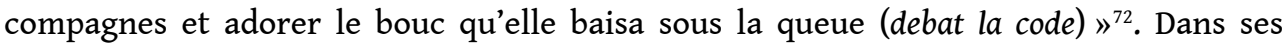
aveux, elle déclare s'être rendue à la maison Iribarnegaray de Juxue, et y avoir ensorcelé avec une poudre une enfant, qui perdit ainsi l'usage d'une jambe. Elle dit aussi avoir usé des mêmes procédés avec son propre fils, qu'elle rendit boiteux et qui mourut peu après, et avoir également délivré son mari de sa marâtre en l'empoisonnant. Malgré ses aveux, Johannette de Sala est condamnée au bûcher.

Le vol nocturne par la cheminée correspond à l'incantation " pet sus fuelha $»^{73}$. Pour se rendre au sabbat, il faut mettre un pied sur la crémaillère et dire " pet sus fuelha ", écrivait déjà le chroniqueur toulousain des Ordonnances du Livre Blanc (1555). Pierre de Lancre cite lui aussi la formule "Pic suber hoeilha, enta la lane de bouc bien m'arrecoueille $»^{74}$, qu'on peut traduire par "Pied sur feuille, ainsi la lande de bouc bien me recueille». Sur le lieu du sabbat, il précise: "communément ils l'appellent Aquelarre, qui signifie lane de bouc, comme qui diroit la lane ou lande où le bouc convoque ses assemblées. Et de faict les sorciers qui confessent, nomment le lieu pour la chose, et la chose ou assemblée pour le lieu, tellement qu'encore que proprement lane de bouc soit le Sabbat qui se tient ès landes, si est-ce qu'ils appellent aussi bien lane de bouc le sabbat qui se tient ès églises, et ès places des villages, paroisses, maisons et autres lieux ».

Avant lui, Estebène de Cambrue, jeune sorcière de la paroisse d'Amou, en 1567, confesse que «le lieu de ceste grande convocation s'appelle généralement par tout le pays la lanne de bouc, où ils se mettent à dancer à l'entour d'une pierre, qui est plantée audit lieu, sur laquelle est assis un grand homme noir ». Et la catalane Narbona Dorcal, en 1498, confesse se rendre au sabbat en utilisant la même formule rituelle : « sur la griffe et sur l'épine, à la lande du bouc $! »^{75}$. Pour se rendre à la « lande de bouc», « diablevauvert " des Pyrénées, les empoisonneuses du Midi frottent de leur onguent la crémaillère de la cheminée et le bâton qu'elles chevauchent. 


\section{Conclusion provisoire}

37 La thèse pyrénéenne de la genèse de la chasse aux sorcières n'est guère soutenue que par l'école anthropologique basque, et la chronologie haute que propose Carlo Ginzburg est largement décriée. Pourtant, si l'on considère le vénéfice comme le «chaînon manquant » des années 1340-1430, on peut imaginer avec Ginzburg qu'« à la lumière de ces documents (les procès contre les «vaudois" piémontais de la seconde moitié du $\mathrm{XIV}^{\mathrm{e}}$ siècle), que Lamothe-Langon ne connaissait pas, les procès inexistants de Toulouse apparaissent comme un "faux critique" singulièrement pénétrant $»^{76}$. Et situer, provisoirement, dans les Pyrénées centrales et occidentales le berceau de la sorcellerie moderne.

\section{NOTES}

1. Un bilan provisoire de ces controverses a été dressé par J.-P. Boudet, « La genèse médiévale de la chasse aux sorcières. Jalons en vue d'une relecture ", Le Mal et le Diable. Leurs figures à la fin du Moyen Âge, Paris, Beauchesne, 1996, p. 34-52.

2. Une bibliographie sommaire, et datée, sur la sorcellerie dans le Midi avait été dressée par J.-F. Le Nail, "Procédures contre des sorcières de Seix en 1562 ", Bulletin de la Société ariégoise des Sciences, Lettres et Arts, 1976, n 31. À travers quelques monographies, quelques sources publiées et les procès du Parlement de Toulouse (1444-1483), et en adoptant la périodisation proposée par R. Kieckhefer, j'arrive, pour le ressort du Parlement de Toulouse, élargi au Béarn, à la Navarre et au Pays Basque, à ces chiffres : pour la démonolâtrie : 1300-1330, 24 procès ; 1331-1375, 4 procès ; 1376-1435, 6 procès; 1436-1499, 19 procès; et pour la sorcellerie : 1300-1330, 14 procès ; $1331-1375,4$ procès ; 1376-1435, 3 procès ; 1436-1499, 75 procès. Au total, 149 affaires, c'est-à-dire à peu près autant que R. Kieckhefer en avait relevé pour la France, la Flandre, l'Italie et l'Angleterre réunies (Alpes non comprises).

3. N. Cohn, Europe's inner demons, New-York, 1975, p. 130, et R. Kieckhefer, European Witch Trials. Their Foundations in Popular and Learned Culture, 1300-1500, Berkeley-Los Angeles, 1976.

4. F. Collard, «Veneficiis vel maleficiis. Réflexions sur les relations entre le crime de poison et la sorcellerie dans l'Occident médiéval », Le Moyen Âge, tome CIX, Paris, 2003, p. 9-57.

5. C. Gauvard, «De grace especial ». Crime, état et société en France à la fin du Moyen Âge, tome 1, Paris, 1991, p. 316. Voir aussi C. Gauvard, "Le Parlement et la sorcellerie au milieu du XV siècle", Finances, pouvoirs et mémoires. Mélanges offerts à Jean Favier, Paris, 1999, p. 85-111.

6. F. Collard, «Venenosa mulier coronata. Variations sur la figure de la reine empoisonneuse dans l'Occident médiéval ", Reines et princesses au Moyen Âge, Cahiers du CRISIMA, 5, tome I, 2001, p. 302-322.

7. C. Gauvard, «Renommées d'être sorcières : quatre femmes devant le prévôt de Paris en 1390-1391 », Milieux naturels, espaces sociaux. Études offertes à Robert Delort, Paris, E.Mornet et Fr. Morenzoni, 1997, p. 703-716.

8. J. Chiffoleau, «Sur la pratique et la conjoncture de l'aveu judiciaire en France du XIII ${ }^{\mathrm{e}}$ siècle au $\mathrm{XV}^{\mathrm{e}}$ siècle ", L'Aveu, Antiquité et Moyen Âge, Actes de la Table Ronde organisée par l'École française de Rome (mars 1984), Rome 1986, p. 370. 
9. N. Gonthier, Délinquance, justice et société dans le Lyonnais médiéval (fin XIIIe-début XVI'), Paris, Le Léopard d'Or, 1993, p. 231.

10. J.-B. Laborde, Les «Brouches » en Béarn, Gascogne et Pays Basque : histoire et folklore, Pau, 1936, p. 49-52.

11. J.-F. Le Nail, «Procédures contre des sorcières de Seix en 1562 », Bulletin de la Société ariégoise des Sciences, Lettres et Arts, 1976, n³1, p. 171, note la même chose : « sur l'assimilation complète du poison à la sorcellerie, on notera que Jehanne de Barbes, victime d'un attouchement maléfique, proprement ensorcelée, déclare avoir été empoisonnée ».

12. Le vocabulaire de la sorcellerie a fait l'objet de nombreuses études, les traductions ici proposées sont largement discutées, la métanalyse lexicalisée le disputant aux étymologies populaires. Les références dépassent le cadre de cette étude, mais seront développées dans ma thèse.

13. E. Leroy-Ladurie, Montaillou, village occitan (1294-1324), Paris, Gallimard, 1975, p. 580-581.

14. Registre d'inquisition de Jacques Fournier (1318-1325), édité par J. Duvernoy, Toulouse, 1965, volume I, p. 243-244.

15. Ibid., p. 247-249.

16. F. Bordes, Sorciers et sorcières. Procès de sorcellerie en Gascogne et en Pays Basque, Toulouse, Privat, 1999.

17. Prosper Dufaur de Larrazet a consacré un article à cette affaire, citée par A. Dupuy, La Lomagne, La Vie d'un pays occitan à travers un millénaire, 16 fascicules, 1990-1995.

18. A. Chassaing, Spicilegium brivatense. Recueil de documents historiques relatifs au Brivadois et à l'Auvergne, Paris, 1886, p. 438-446.

19. J. Artières, Documents sur la ville de Millau. Mémorial des privilèges, livres de comptes des consuls boursiers et délibérations communales ( $\mathrm{XI}^{e}-\mathrm{XVI}{ }^{e}$ siècles), Archives Historiques du Rouergue, tome VII, Millau, 1930.

20. Archives Départementales de Haute-Garonne, B2309, jeudi 12 février 1461. Désormais, registres d'arrêts et d'audiences du Parlement de Toulouse ne sont cités que par leurs cotes. Signalons que trois autres agents du comte d'Armagnac sont poursuivis pour de semblables insultes en 1468, en 1474 et en 1484.

21. B2299, mardi 21 février 1447.

22. B2306, mardi 26 juillet 1456 .

23. B2306, mardi 8 février 1457.

24. B2322, mardi 7 juillet 1478 .

25. B2322, jeudi 25 juin 1478 .

26. Ces procès des sorcières de Montaigut-en-Combraille, Beaumont (B2306), Lichemialle (B2307) ou Tournon-sur-Rhône (B2308), qui éclatent dans les années 1451-1455, sont pourtant directement liés à la présence du fameux Guillaume Adeline et à l'activité de l'inquisiteur Nicolas Jacquier, convaincu de l'alliance infernale des maléfices et poisons.

27. B2301, mardi 13 août 1450.

28. B2308, mardi 15 juillet 1460 .

29. B2312, mardi 12 février 1465.

30. B2319, jeudi 3 mars 1474 .

31. B2322, jeudi 4 décembre 1477.

32. B2320, mardi 7 mars 1475 .

33. C. Ginzburg, Le Sabbat des sorcières, Paris, 1992, p. 54.

34. N. Ghersi, "Tragique expédition punitive contre Katherine, la sorcière de Béziers (1440) ", Heresis, $\mathrm{n}^{\circ}$ 42-43, Carcassonne, 2005, p. 101-120.

35. Registres DDD et GGG de l'Inquisition de Carcassonne (1325-1329), édités par J. Duvernoy, E. Jolibois, «Le procès de la sorcière brûlée à Labruguière en 1485 ", Revue du Tarn, tome 1, 1876, et A. Charnay, «Médecine empirique et remèdes diaboliques: les secrets de Peyrona Galiberta, 
sorcière de Labruguière ", Bulletin de la Société des Sciences, Arts et Belles-Lettres du Tarn, 1998, n50-51, p. 69-78.

36. P. Braun, "La sorcellerie dans les lettres de rémission du Trésor des Chartes ", Études sur la sensibilité au Moyen Âge, Actes du 102ème Congrès des Sociétés Savantes (Limoges, 1977), Paris, 1979, p. 267.

37. J.-F. Le Nail, « Procédures contre des sorcières de Seix en 1562 », Bulletin de la Société ariégoise des Sciences, Lettres et Arts, 1976, n³1, p. 171.

38. B2320, mardi 7 mars 1475.

39. Registre d'inquisition de Jacques Fournier (1318-1325), édité par J. Duvernoy, Toulouse, 1965, volume II, p. 69.

40. Archives Nationales, JJ 196, $n^{\circ} 127$, fol.72, et JJ 197, $n^{\circ} 104$, fol. 62.

41. On impose à Gailhardine Dailhet six ou sept chefs d'accusation, «mais n'y a tesmoing qui en depose de perfecta sciencia, sinon de ung ou deux qui dient avoir esté empousonnez par elle et autres en y a qui parlent XIII ou XIIII ans ». Lauret donne quelques précisions: "dit que le premier cas ou imponitur est que XIIII ans a, elle empousonna Jehanne, femme de Domenge de Pini, en une fogasse chinilde trampée en vin, qu'elle lui bailla à manger et que ladite Jehanne est depuis morte, sur quoy a ung tesmoing, c'est assavoir le mary de ladite Jehanne, qui depose que ung mege le lui dist, mais ne declaire quelle pouson ». Lauret « dit que le second cas est que trois ans a, ledit appellant empousonna Jamet de La Fossio, avecques une souppe en vin et que s'il n'eust vomy il en feust mort, et que apres doubtant la justice, elle lui donna poculum contrarium, et cecy depose ledit Jamet mesme, et hoc diversi modo, car es informacions il depose qu'elle estoit diffamée d'estre pousonniere, et en l'enqueste dit que non et aussi qu'il ne vomy point ».

42. Registre d'inquisition de Jacques Fournier (1318-1325), édité par J. Duvernoy, Toulouse, 1965, volume II, p. 388, et A. Charnay, "Un sorcier à Cordes en 1344 ? Le procès de Bernard Fort ", Bulletin de la Société des Sciences, Arts et Belles-Lettres du Tarn, n 49, 1995, p. 477-496.

43. On relève devant le Parlement de Toulouse (1444-1483) vingt-huit affaires d'empoisonnement sans lien avec la sorcellerie telle que nous l'avons définie, bien que l'expression «fors! fors!", relevée aussi par Franck Collard, et le contexte (affaires Jacques Cœur et Otto Castellani, qui combinent nigromancie et crimes crapuleux) soulignent le caractère imparfait de ces classifications.

44. Voir par exemple le procès très documenté de Rique de Santon et de Pierre Puy, qui débute B2302, mardi 19 janvier 1451.

45. B2320, mardi 7 mars 1475 : «par delà vint ung grand esclandre, car y avoit hommes qui latrabant ut canes, autres qui ululabant ut lupi, autres qui garriebant ut corni, et autres qui venoient en dessiechement et finablement en mouroient ».

46. Les sorcières de Gourdon-sur-Lot, au début du XIV ${ }^{\mathrm{e}}$ siècle, agissent déjà ainsi. Guilherma Robberta invoque le diable en dessinant un cercle, dans lequel elle se tient avec un enfant et en portant un chaudron où elle jette du plomb, et en disant "Bausabut et Barraban et Lucifer lucifian ». Grâce à son art, elle trouve et brûle un paquet maléfique, qui contient ongles, poils, plomb, serpents et crapauds, sous le seuil de la porte d'un boucher dont les enfants mouraient les uns après les autres. Guirauda de L'Estanc invoque aussi Barraban, mais c'est surtout une empoisonneuse, qui fabrique ses potions avec de la pervenche, du céleri, des os de morts déterrés au cimetière et du pain bénit. Accusée de vénéfice contre une enfant, Bertranda de Gencelias, elle conjure le sort: les envoûteurs sont aussi d'efficaces désenvoûteurs. Voir A. Charnay, «Sept sorcières de Gourdon au début du XIV ${ }^{\mathrm{e}}$ siècle ", Bulletin de la Société des Études du Lot, tome CXV, 1994, p. 17-50, avec en annexe, traduction de la langue d'oc des différents procès (Archives Municipales de Gourdon, FF 11 et FF 12). Sur le sabbat, voir infra et J. Frayssenge, « Le sabbat des sorcières. La répression de l'hérésie sorcellaire à Millau au $\mathrm{XV}^{\mathrm{e}}$ siècle », Heresis, $\mathrm{n}^{\circ}$ 44-45, Carcassonne, 2006, p.189-206. 
47. Certes, le choix de distinguer aussi nettement invocateurs de démons et empoisonneuses est discutable, mais même en tenant compte des magiciens, le résultat obtenu est limpide : 42 «sorciers" pour 169 sorcières dans le Midi. À l'inverse, vu l'importance du vénéfice dans les accusations de sorcellerie, on aurait pu intégrer dans les mêmes statistiques toutes les « empoisonneuses ", aussi nombreuses que les sorcières, et dont le procès ne nous est parfois pas parvenu en détail.

48. «Le seigneur de Leran avoit fait prandre un homme et deux femmes pouçonnieres».

49. «Cependant advint que à Leran avoit deux sorcieres prinses».

50. « Pour raison de certain proces par lui receu en la dite court de Leran, c'est assavoir touchant trois femmes qui y ont esté brulées ».

51. P. Braun, «La sorcellerie dans les lettres de rémission du Trésor des Chartes », Études sur la sensibilité au Moyen Âge, Actes du $102^{\text {ème }}$ Congrès des Sociétés Savantes (Limoges, 1977), Paris, 1979, p. 257-278.

52. C. Gauvard, «Renommées d'être sorcières: quatre femmes devant le prévôt de Paris en 1390-1391 ", Milieux naturels, espaces sociaux. Études offertes à Robert Delort, Paris, E.Mornet et Fr. Morenzoni, 1997, p. 703-716.

53. J.-F. Le Nail, «Procédures contre des sorcières de Seix en 1562 », Bulletin de la Société ariégoise des Sciences, Lettres et Arts, 1976, $\mathrm{n}^{\circ}$ 31, p. 163.

54. Paul Ourliac et Jean-Louis Gazzaniga, Histoire du droit privé français, de l'an mil au code civil, Paris, 1985.

55. La transcription de ces procès prendrait plusieurs volumes. Pour les premières années du «dossier » Lévis-Mirepoix, B1, 17 décembre 1445, 20 décembre 1445, 22 décembre 1445, $1^{\text {er }}$ février 1446, 19 mars 1446, 23 mars 1446, 26 mars 1446, 31 mars 1446, 2 mai 1446, 9 juillet 1446, 21 juillet 1446, 15 mai 1447, 20 mai 1447, 24 mai 1447, 15 juillet 1447, 17 avril 1448, 10 juillet 1448, 12 septembre 1448, 13 septembre 1448, 16 septembre 1448, 4 avril 1449, 14 mai 1449, 16 mai 1449, 2 juillet 1449, 16 août 1449, 18 septembre 1449, 27 novembre 1449, 29 décembre 1450, 6 février 1451, 16 mars 1451, 3 avril 1451, 7 mai 1451, 2 juin 1451, 29 juillet 1451, 21 août 1451, 23 août 1451, 16 février 1452, 21 avril 1452, 9 mai 1452, 17 août 1452, 28 août 1452, $1^{\text {er }}$ septembre 1452 , etc. B2298, 10 mai 1446, 9 juin 1446, 13 juin 1446, 20 juin 1446, 4 juillet 1446, 7 juillet 1446. B2299, 31 janvier 1447, 22 mai 1447, 25 mai 1447, $1^{\text {er }}$ juin 1447, 6 juin 1447, 13 juin 1447. B2300, 5 décembre 1448, 2 janvier 1449, 9 janvier 1449, 23 janvier 1449, 27 janvier 1449, 27 mars 1449, 28

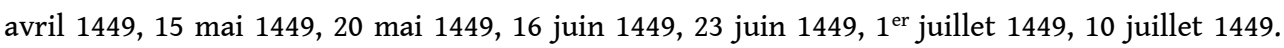
B2302, 25 janvier 1451, 18 mars $1451,1^{\text {er }}$ avril 1451, 10 mai $1451,1^{\text {er }}$ juin 1451,5 juillet 1451,27 juillet 1451. B2303, 4 décembre 1452, etc. Pour les premières années du "dossier » LévisFlorensac, B1, 17 juillet 1445, 24 juillet 1445, 4 septembre 1445, $1^{\text {er }}$ avril 1446, 24 mai 1447, 8 juillet 1447, 26 janvier 1448, 16 mars 1448, 23 avril 1448, $1^{\text {er }}$ juillet 1449, 3 mars 1451, 16 mars 1451, 4 septembre 1452, 16 septembre 1452, etc. B2297, 31 décembre 1444, 5 janvier 1445, 12 janvier 1445, 10 juin 1445, 14 juin 1445. B2298, 21 mars 1446, 16 mai 1446, 20 juin 1446, 21 juin 1446, 27 juin 1446. B2299, 5 juin 1447, 26 juin 1447, 24 juillet 1447. B2300, 5 août 1449, 7 août 1449, 11 août 1449. B2301, 27 janvier 1450, 12 mai 1450, 18 juin 1450. B2302, 14 janvier 1451, 28 janvier 1451, 11 février 1451,15 mars $1451,1^{\mathrm{er}}$ avril 1451, 3 mai 1451, 4 mai 1451,17 mai 1451, 20 mai 1451. B2304, 7 janvier 1454 , etc.

56. B2299, jeudi 25 mai 1447 : «qu'elle est belle, on le voit, de la bonté, on se rapporte à ce qu'il en est, est vray qu'elle est une des subtiles femmes de Languedoc et par aventure plus que n'est mestier à une femme ".

57. Jean de Lévis suggère que le médecin a pu être complice du crime. B2299, jeudi $1^{\text {er }}$ juin 1447 : «Maillorque fist venir ung medicin de son pais qui demouroit à Carcassonne, lequel en pourroit aussi bien savoir quelque chose, car il tint la main à vendre les taces que la demanderesse avoit prins en l'ostel apres la mort de son dit mary ». 
58. B2298, lundi 20 juin 1446 :» il fut fame au pais que on luy avoit anticipé ses jours par poisons ». B2299, jeudi 25 mai 1447 : "incontinant que le defendeur fut venu à l'ostel, fame publique fut que son dit frere estoit mort de poisons et que on lui avoit anticipé ses jours et commota sunt viscera eius ".

59. Jean Genoyn, lieutenant du viguier de Béziers et meurtrier de la « sorcière-empoisonneuse » Katherine Coterigue, a participé à ce procès.

60. Le mot «hantauma " désigne encore la sorcière en Languedoc. "Avoir les territoires " signifie pouvoir juger hors de ses terres, sans transport de justice et avec l'accord du seigneur haut-justicier lésé. Ici, Gaston de Léran consent que Jean de Lévis et ses gens interrogent le sorcier et les sorcières qu'il tient en prison.

61. Eustache de Mirepoix, qui a livré Maillorque à la justice, défend donc Aelips, et indirectement les sorcières.

62. S'agit-il du temps de Roger-Bernard II (mort en 1419) ou de Philippe II (mort en 1442)? L'absence de procès laisse imaginer que c'est pure invention, mais entre 1422 et 1425, il y a des bûchers contre les sorcières en Armagnac, à Millau, à Toulouse et dans le Val d'Aneu.

63. La sorcière est aussi prophétesse : quand Philippe était à la guerre, on avait demandé à la Gausagne s'il en reviendrait. Elle aurait répondu « que oil mais il ne vivroit gueres ».

64. J. Kelly, Women, history, and theory, Chicago-London, University of Chicago Press, 1984, et A.M. Lucas, Women in the Middle Ages. Religion, marriage and letters, Brighton, Harvester Press, 1983.

65. La thèse que j'ai entreprise sous la direction de Claude Gauvard porte sur « La culpabilité des femmes au Parlement de Toulouse, 1444-1483 » et développe plus amplement ce sujet.

66. C'est en 1409 que le pape Alexandre V évoque pour la première fois la « secte » des sorciers qui complote contre la Chrétienté.

67. C. Lison Tolosana, Brujeria, estructura social y simbolismo en Galicia, Madrid, 1983, p. 28, traduit aux Presses Universitaires de France, Paris, 1994. Voir aussi J.-J. Saroïhandy, « El boque de biterna en los fueros catalanes del valle de Aneu », Revista de Filología Española, 1917, tome IV, p. 26-49, p. 33, et C. Gomis i Mestre, La Bruixa catalana, réédition, Barcelone, Alta Fulla, 1987, p. 208-209.

68. A. Gari Lacruz, « La sorcellerie dans les Pyrénées centrales à l'époque moderne », Tolérance et solidarités dans les pays pyrénéens, Actes du colloque de Foix sur le Quatrième centenaire de l'Edit de Nantes, Foix, Archives de l'Ariège, 2000, « Les Sabbats en Aragon d'après les documents et la tradition orale ", Sabbat des Sorciers en Europe: XVe-XVIII siècles, Paris, Jérôme Millon, 1993, et «Los aquelarres en Aragón según los documentos y la tradición oral», Temas de Antropologia Aragonesa, n4, 1993, 241-261.

69. Lors d'un procès, il est rapporté qu'à l'entrée du roi Charles VII à Toulouse, le 8 juin 1442, le sénéchal libéra des sorcières (B2309, mardi 20 janvier 1461).

70. B2299, mardi 28 février 1447, et B2299, mardi 13 juin 1447.

71. N. Cohn, Démonolâtrie et sorcellerie au Moyen Âge. Fantasmes et réalités, Paris, Payot, 1982, p. 266.

72. Abbé Larramendy (curé de Garris, sous le pseudonyme Landemont), « Procès de sorcellerie en Basse-Navarre ", Revue de Béarn, Navarre et Lannes, tome I, 1883, p. 49-54, cité par F. Bordes, Recherches sur la sorcellerie dans le Béarn, les Landes et le Labourd sous l'Ancien Régime, Thèse de l'École des Chartes, 1978.

73. J.-P. Piniès, « Pet-sus-fuelha ou le départ des sorcières pour le sabbat », Heresis, ${ }^{\circ}$ 44-45, 2006, Carcassonne, p.247-266.

74. Pierre de Lancre, Le Tableau de L'inconstance des Mauvais Anges et Démonsoù il est amplement traité des sorciers et de la sorcellerie, Paris, Nicolas Buon, 1613, livre II, discours 4.

75. En Aragon, les ethnologues modernes signalent cette formule : « sobre arto y sobre espina, a las Lannas del Boch ayna! ».

76. Carlo Ginzburg, Storia notturna. Una decifrazione del sabba, Turin, Einaudi, 1989, Le Sabbat des sorcières, Paris, 1992, p. 308, note 94. 


\section{RÉSUMÉS}

Les procès de sorcellerie dans le Midi de la France présentent dès le début du $\mathrm{XV}^{\mathrm{e}}$ siècle des élémentss qui font de ces contrées un candidat sérieux dans l'introuvable genèse de la chasse aux sorcières: précocité $\mathrm{du}$ sabbat, caractère féminin $\mathrm{du}$ crime, importance $\mathrm{du}$ veneficium (empoisonnement par sortilège). L'omniprésence du poison fait d'ailleurs le lien avec les grands procès de démonolâtrie d'après les temps cathares. Ces procès constituent aussi un autre chapitre de l'histoire des femmes, dans un contexte de radicalisation des sermons et de destabilisation des maisons de Foix-Béarn, Comminges et Armagnac.

The fifteenth-century witchcraft trials in southwestern France present some characteristics that make them one of the best milestones in the undefinableorigins of witchhunts: the early appearance of the witches' sabbath, the predominantly female nature of the crime, the importance of veneficium (poisoning by spell). The prominence of poison links these trials with the major demonolatry trials in the years after Catharism. These trials constitute another chapter in women's history in the context of the radicalisation of sermons and the destabilisation of the Houses of Foix-Bearn, Comminges and Armagnac.

\section{AUTEUR}

\section{NICOLAS GHERSI}

Université Paris-I. 